\title{
BMJ Open Instruments measuring the quality of life among people living with type 2 diabetes mellitus in India: a systematic review protocol
}

Chetna Demla, ${ }^{1}$ Anns Thomas, ${ }^{1}$ Jomol Jose, ${ }^{1}$ Angela L Joshy, ${ }^{1}$ M A Hrishikesh, ${ }^{1}$ Ambigai Rajendran, ${ }^{1}$ Shradha S Parsekar (D) ${ }^{2}$

To cite: Demla C, Thomas A, Jose J, et al. Instruments measuring the quality of life among people living with type 2 diabetes mellitus in India: a systematic review protocol. BMJ Open 2021;11:e043831. doi:10.1136/ bmjopen-2020-043831

- Prepublication history for this paper is available online. To view these files, please visit the journal online (http://dx.doi. org/10.1136/bmjopen-2020043831).

Received 14 August 2020 Revised 10 March 2021 Accepted 24 March 2021

Check for updates

(C) Author(s) (or their employer(s)) 2021. Re-use permitted under CC BY-NC. No commercial re-use. See rights and permissions. Published by BMJ.

${ }^{1}$ Department of Commerce, Manipal Academy of Higher Education, Manipal, Karnataka, India

${ }^{2}$ Public Health Evidence South Asia, Department of Health Information, Prasanna School of Public Health (PSPH), Manipal Academy of Higher Education, Manipal Academy of Higher Education, Manipal, Karnataka, India

Correspondence to Dr Shradha S Parsekar; shradha.parsekar@manipal.edu

\section{ABSTRACT}

Introduction Type 2 diabetes mellitus affects an individual's quality of life (QoL); and there are multiple instruments that can be used to measure QoL. The purpose of this systematic review is to identify the existing instruments that have been used to measure QoL in people living with diabetes, and to enlist the major domains (such as physical and psychological components) available in the identified instruments. Additionally, we plan to determine the psychometric properties of the identified QoL instruments using COnsensus-based Standards for the selection of health Measurement INstruments (COSMIN) methodology.

Methods and analysis The Preferred Reporting Items for Systematic Reviews and Meta-Analyses Protocol guideline was followed to report this systematic review protocol. Searches will be conducted on MEDLINE (via PubMed, Web of Science), SCOPUS and CINAHL. Predetermined inclusion/ exclusion criteria will be applied to the search results, to include studies with adult individuals diagnosed with type 2 diabetes mellitus, with and without complications, and exclude studies with type 1 diabetes or other clinical illness. Studies conducted outside India will be excluded. Five authors in pairs will independently screen the articles and extract the data that meets the inclusion criteria. The COSMIN criteria will be used to assess the risk of bias of included studies. Narrative synthesis will be performed to analyse the findings of the instruments.

Ethics and dissemination Ethical permission is not applicable, as this is a systematic review. We intend to disseminate the systematic review findings through a national or international conference and publish the findings in a peer-reviewed journal.

PROSPERO registration number CRD42020180432.

\section{INTRODUCTION}

'Type 2 diabetes mellitus' is one of the noncommunicable diseases that occurs when the pancreas is unable to produce required amount of insulin, or when the body cannot use insulin appropriately. The risk of type 2 diabetes rises with increasing age and therefore, it is diagnosed mostly in the middle and older aged population. ${ }^{1}$ However, recently
Strengths and limitations of this study

Systematic review proposed to identify and summarise the disease-specific and generic instruments used in India for assessing the quality of life of individuals with type 2 diabetes.

- COnsensus-based Standards for the selection of health Measurement INstruments methods will be followed to assess the psychometric properties.

- We anticipate heterogeneity in population, context, diabetes-related complications, types of instruments, domains/themes being measured and study methods.

its incidence is found to be intensifying among youth and children. ${ }^{1}$ Initial signs of type 2 diabetes may include repeated urination, increased appetite and thirst, blurred vision, fatigue and sluggish healing of cuts and wounds that may remain unnoticed. As a result of lack of awareness of the initial symptoms / signs compounded with other demandside and supply-side factors, type 2 diabetes may be diagnosed many years after its onset viz. the stage when the disease has progressed to complications.

Diabetes is a worldwide epidemic, with the possibility to cause an overall healthcare emergency. It has been anticipated that by 2025 , approximately 300 million individuals would be affected by it. ${ }^{2}$ About 50.9 million resident Indians experience ill effects of diabetes, and by 2025 . India will be the diabetes capital of the world with an estimated 80 million diagnosed with the disease. ${ }^{2}$ India already has the maximum estimated number of people diagnosed with diabetes globally (approximately 73 million) that indicates every fifth individual with diabetes in the world belongs to India. ${ }^{3}$ In 2006, there were about 40.9 million resident Indians with diabetes, out of which $90 \%$ were type 2 diabetes, with varying prevalence 
in rural $(2.4 \%)$ and urban $(11.6 \%)$ regions. ${ }^{4}$ There are multiple factors responsible for the growing burden of type 2 diabetes viz. unhealthy diets, sedentary lifestyles, rapid urbanisation, substance use, improvement in life expectancy, ${ }^{1}$ excess body weight ${ }^{5}$ and genetic predisposition. $^{2}$ Growing cases of diabetes are straining the presently weak healthcare sector and economy of India. ${ }^{6}$

It is now well established that type 2 diabetes affects the quality of life (QoL) of an individual. ${ }^{7}$ WHO (2019) defines QoL as; 'an individual's perception of their position in life in the context of the culture and value systems in which they live and in relation to their goals, expectations, standards and concerns'. QoL encompasses a person's overall health, opinions, social interactions and association to various characteristics of one's environment, thus, making it a multidimensional concept. ${ }^{8}$ The 'health-related QoL' (HRQoL) has been defined within the WHO QoL system as those aspects of QoL that affect a person's physical and mental well-being. ${ }^{9}$ Considering the importance of QoL, stakeholders across the world are increasingly paying attention to the disease-specific QoL of an individual and trying to optimise QoL within the healthcare budget. In such a situation, it becomes imperative to assess the HRQoL or QoL. Developing a robust understanding of HRQoL or QoL among individuals living with type 2 diabetes would help the healthcare providers to offer effective interventions and treatment choices that minimise the negative impact of diabetes on QoL.

Fitzpatrick et al have developed the criteria to assess the standard HRQoL measure and have reported the following important issues in measuring HRQoL; whether it is objective or subjective, generic or disease specific, its dimensionality, reported by self or proxy, measurement properties and the HRQoL selection criteria. ${ }^{10}$ Assessment of validity and reliability of instruments that is, psychometric properties, helps in understanding the quality of the instruments. Thus, awareness of psychometric or measurement properties of the instrument enables the researchers and clinicians to select the appropriate instrument for their research and clinical practice, respectively. The psychometric properties of the instrument include reliability and validity. 'Reliability is the capacity to replicate a consistent result in time and space, or from different observers, with aspects relating to coherence, consistency, equivalence and homogeneity'. ${ }^{11}$ Validity means an instrument tests exactly what it aims to measure. ${ }^{12}$

Studies, ${ }^{2}{ }^{13-16}$ that investigate the QoL of individuals with type 2 diabetes and the associated factors, have been conducted previously in India. It is challenging to summarise the results of these studies as they used different QoL instruments. The QoL of an individual is influenced by the severity of the disease condition, culture $^{17}$ and other demand-side and supply-side factors. Furthermore, the high financial burden ${ }^{18}$ associated with chronic conditions such as diabetes in resource limited countries (like India) may have a larger effect on QoL.
As a result, the instruments validated elsewhere may not be able to adequately capture QoL for individuals living in India. Nevertheless, to the best of our knowledge, no systematic reviews have appropriately assessed the psychometric properties of available QoL measures among people living with type 2 diabetes mellitus in India.

Considering the availability of a number of generic and disease-specific questionnaires, it is a challenge to select the most appropriate patient-reported outcome measures (PROMs). ${ }^{17}$ An ideal PROM should be sensitive for changes, provide information about disease-specific associations with construct being studied, enable comparison between various groups of patients or general population and be useful in economic evaluation. ${ }^{19}$ Therefore, this systematic review is aimed at identifying the existing instruments that have been used to measure QoL in people living with diabetes in India. As the QoL is a multidimensional construct, we will enlist the major domains or themes (may include physical, psychological and social components) of the identified instruments. Additionally, we will determine the psychometric properties (content and structural validity) of the identified QoL instruments using 'COnsensusbased Standards for the selection of health Measurement INstruments' (COSMIN) methodology. This review will be helpful for researchers, decision makers and economists in selecting appropriate instruments to measure QoL among people living with type 2 diabetes mellitus in India

\section{METHODOLOGY}

We followed the 'Preferred Reporting Items for Systematic Reviews and Meta-Analyses Protocols (PRISMA-P) 2015 guidelines' to report this systematic review protocol. ${ }^{20} \mathrm{We}$ will use the 10-step procedure to carry out this systematic review as suggested in COSMIN methodology. ${ }^{21} 22$

\section{Inclusion and exclusion criteria of studies \\ Participants}

Adult $(>/=18$ years $)$ individuals having type 2 diabetes mellitus (diagnosed by medical practitioner and/or confirmed by laboratory reports) in India (with and without complications), will be included, irrespective of the duration of diabetes. In the case of mixed population, we will consider studies reporting subgroups of population of our interest. We will exclude individuals with type 1 diabetes mellitus, gestational diabetes, life-threatening illness and those who are terminally ill. Type 2 diabetic individuals with other chronic communicable diseases such as HIV and tuberculosis will be excluded. Studies conducted in countries other than India, with population of Indian origin/ descent, will be excluded. Caregivers acting as a proxy for the diabetic individuals to measure the QoL or studies assessing caregiver QoL will not be considered as we want to restrict to the patient reported QoL.

\section{Study design}

Studies related to QoL instrument development and/or measurement of psychometric properties will be included. 


\section{Outcomes}

The primary outcome is to identify all PROMs that have been used to assess QoL in studies of individuals living in India with type 2 diabetes mellitus. The QoL instruments could be disease specific (eg, Quality of Life Instrument for Indian Diabetes patients (QOLID)) ${ }^{13}$ or generic QoL (eg, EuroQol-5 Dimension (EQ-5D), Short From Health Survey (SF-36)). We do not impose a strict definition of QoL and therefore, we will not restrict the inclusion depending on definition of QoL. Considering the multidimensionality of QoL, a study will be eligible to be included if it has measured/ scored a specific domain(s)/ construct(s) (eg, psychological component) of QoL as a unidimensional measure. The QoL instruments that was developed in other countries are eligible to be included, if it has been validated among resident Indian population living with type 2 diabetes. However, in this case (if required), we will refer to the original PROM development study to look for content of the instrument. Additionally, we will critique measurement properties of identified instruments as measured by the studies. The psychometric or measurement properties in this systematic review will include content validity and internal structure as defined by the COSMIN study. ${ }^{23-26}$ Further, justification and description of psychometric properties can be found below under the 'risk of bias (RoB) assessment of included studies'. We will also report major domains or themes (such as physical or psychological components) of the identified QoL instruments

\section{Search strategy}

We will undertake a comprehensive and sensitive electronic database search on MEDLINE (via PubMed, Web of Science), SCOPUS and CINAHL. Each database will be searched from its inception to March 2021. We will use search terms based on Medical Subject Heading and free-text search terms, and the sensitive search strategy suggested by Terwee $e t a l^{27}$ will be followed. The major search concepts will be, QoL (construct), diabetes mellitus (population) and instruments. Furthermore, we will apply the measurement property filter as suggested in the COSMIN study. ${ }^{27}$ The search concepts and keywords are listed in table 1. Additionally, we will conduct a reference search and forward citation tracking of the included studies to identify potential records. A designated information scientist will run the search in abovementioned databases. The search will be restricted to studies published in English language. The initial search will be undertaken in PubMed, which will be customised for other databases. The results will be exported to reference manager software where the duplicates will be eliminated. The citations will then be exported to an Excel spreadsheet for screening.

\section{Screening and selection of studies}

Five authors (CD, ALJ, AT, JJ and MAH) in groups of two will begin with title screening independently. In case of disagreements for inclusion or exclusion of studies,

\begin{tabular}{|c|c|}
\hline Construct & $\begin{array}{l}\text { 'quality of life', 'QOL', 'Qol', 'HrQoL', 'HRQL' } \\
\text { 'Health related quality of life', 'Quality } \\
\text { of life instrument for diabetes patients', } \\
\text { 'QOLID', 'EQ-5D', 'SF-36', 'short form 36', } \\
\text { 'WHO quality of life Scale-Brief Version', } \\
\text { 'WHOQOL-BREF', 'WHO quality of life', } \\
\text { 'ADDQoL', 'The Audit of Diabetes Dependent } \\
\text { Quality of Life', Questionnaire for Life } \\
\text { Instrument for Indian Diabetes Patients', etc. }\end{array}$ \\
\hline Instrument & $\begin{array}{l}\text { 'Instruments', 'Tools', 'Measures', } \\
\text { 'Questionnaire', 'Score', 'Patient-reported } \\
\text { outcome measures', 'PROMs', 'Outcome } \\
\text { assessment', 'Outcome score', 'Scale' etc. }\end{array}$ \\
\hline Population & $\begin{array}{l}\text { 'Diabetes Mellitus', 'Diabet’, 'T2DM', } \\
\text { 'NIDDM', 'Glucose intolerance', } \\
\text { 'Hyperglycaemic', 'Hypoglycaemic', } \\
\text { 'metabolic diseases', 'type-2 diabetes' }\end{array}$ \\
\hline $\begin{array}{l}\text { Measurement } \\
\text { properties }\end{array}$ & $\begin{array}{l}\text { COSMIN measurement properties filter }{ }^{27} \\
\text { AND exclusion filter }\end{array}$ \\
\hline Country & Country specific filter (India) \\
\hline
\end{tabular}

a discussion will be held until consensus is achieved. In case a consensus is not achieved, we will contact the third author (SSP). The third author will act as an arbitrator and final decision maker. We will follow the same process to screen the abstracts and full text. We anticipate that our search will yield a large number of PROM, therefore, to screen the appropriate studies we will use a screening protocol as documented in table 2 . We will describe the reasons for exclusion for all studies excluded by the assessors. The screening process will be documented using the PRISMA flow diagram.

\section{Data extraction}

The data collation will be undertaken independently by five authors (CD, ALJ, AT, JJ, MAH) in groups of two, to minimise errors and reduce potential biases. Any disagreement will be resolved by having a discussion within and between the groups or contacting the third author (SSP) for a final decision. We will create a structured data collation form to obtain all relevant information from each included study. The data extraction form will be initially pilot-tested by all the authors for two to three studies. Table 3 enlists the details that we will extract from each of the included studies. In case of missing data or incomplete information in the study, the corresponding author will be contacted. If there is no reply from the corresponding author after waiting for 15 days, the study will be excluded.

\section{RoB assessment of included studies}

We will apply the COSMIN RoB checklist to assess the methodological quality of the included studies. ${ }^{2428} 29$ The COSMIN study does not dictate the use of overall checklist and recommends that assessment of each measurement property can be considered as a distinct study. 
Table 2 Screening protocol

\section{Title and abstract screening}

A Is the study written in English language and published after $1990 ?$

B Is it a study using QoL instrument? OR

Is it a QoL instrument development study or study measuring psychometric properties?

Are you in doubt?

C Is the study based on QoL of type 2 diabetes mellitus in India? AND

Do the participants under study are above or equal to 18 years?

Are you in doubt?

D Does the study mention generic or disease-specific QoL instrument for type 2 Diabetes? OR

Does the study include psychometric property such as validity or reliability for measuring standards of QoL instrument? Are you in doubt?
If answer to both the components is 'yes', then go to $B$

\section{If answer is 'yes' for either}

one or all the components, then go to $\mathrm{C}$.
If not, exclude the study

If not, exclude the study.

If 'yes', then go to D. If not, exclude the study.

2 Full-text screening

$1 \mathrm{~A}$ Study design

Is it a survey or case study using QoL instruments for type 2 diabetes patients? OR

Is it a study related to QoL instrument development? OR Is it a study measuring psychometric properties?

1B Check for multiple publications of the same study. We will include all publications but include the most extensive one.

2 Population

1. Does the population under study have type 2 diabetes mellitus (diagnosed by a medical practitioner and/or confirmed by laboratory reports) in India, with or without complications?

2. Does study consider adults ( $>$ or $=18$ years) population?

3. Does it involve a mixed population, but have reported subgroups of population of our interest?

\section{Outcomes}

1. Studies assessing generic or disease-specific instruments for individuals diagnosed with type 2 diabetes mellitus.

2. Studies measuring a specific domain(s)/construct(s) (eg, psychological) of QoL.

3. Studies specifying psychometric characteristics such as reliability and validity (defined by COSMIN) of identified instruments as measured by the studies.

4. Studies reporting dimensions or domains of the identified QoL instruments.

Note: The QoL instrument(s) that are developed in other countries are eligible to be included if the studies have validated it among Indian population.
If the answer to all is 'yes', then go to point no. 2. Go to a point no. 1B for more details

If the answer to any one is Yes, then go to point no. 2 .

If the answer to ' $a$ ' and ' $b$ ' is Exclude based on population if none of the Yes, then go to point no: 3 . options are applicable. If you are in doubt in case Exclude adults with type one diabetes of point ' $c$ ' please mark the mellitus, gestational diabetes, lifestudy for discussion.

If the answer to ' $a$ ' is Yes, then include the study. Additionally, if answer to ' $b$ ' or ' $c$ ' is Yes then include the study.
Exclude the study based on study design: if none of the options are applicable. terminally ill, diagnosed with HIV or tuberculosis.

Caregivers acting as a proxy for the diabetic individual to measure the Qol or studies assessing caregiver Qol will not be considered.

Exclude if the study has been conducted outside India, irrespective whether the population were of Indian origin.

\section{Exclude study based on outcome if none of} the mentioned outcomes are assessed.

COSMIN, COnsensus-based Standards for the selection of health Measurement INstruments ; QoL, quality of life.

Therefore, for the purpose of this systematic review, we will restrict the checklist to assess content validity and internal structure. This corresponds to five checklists of COSMIN RoB checklist: PROM development (35 items), content validity (31 items), structural validity (4 items), internal consistency (5 items) and cross-cultural validity (4 items). ${ }^{23-2528}$ The content validity echoes the construct that is supposed to be measured and it can affect other measurement properties of the instrument. Therefore, assessment of content validity is essentially the first step of measurement of the validity of a PROM. Overall judgement for content validity will be decided based on 4-point ratings ('very good, adequate, doubtful and inadequate'). To assess the final result, the lowest score that is, "worst 


\begin{tabular}{|c|c|}
\hline $\begin{array}{l}\text { Article } \\
\text { information }\end{array}$ & $\begin{array}{l}\text { First author and year of publication } \\
\text { Title } \\
\text { Country of origin } \\
\text { Citation details } \\
\text { Study period }\end{array}$ \\
\hline $\begin{array}{l}\text { Instrument } \\
\text { details }\end{array}$ & $\begin{array}{l}\text { Name of the instrument } \\
\text { Outcome measure: generic or disease } \\
\text { specific } \\
\text { Timing of administration } \\
\text { Instrument use } \\
\text { Language used } \\
\text { No and type of dimensions } \\
\text { No of items } \\
\text { Scale design } \\
\text { Population information: stage of illness, } \\
\text { types of complications (if any), age, } \\
\text { gender, ethnicity, place of residence, } \\
\text { setting } \\
\text { Other contextual factors such as region }\end{array}$ \\
\hline $\begin{array}{l}\text { Psychometric } \\
\text { characteristics }\end{array}$ & $\begin{array}{l}\text { Validity } \\
\text { Theoretical/conceptual framework } \\
\text { Type of validity tests conducted and } \\
\text { results } \\
\text { Reliability } \\
\text { Type of tests conducted and results } \\
\text { Statistical response } \\
\text { Sample size } \\
\text { Content validity outcomes } \\
\text { Time required to complete the instrument } \\
\text { Mode of administration (ie, self- } \\
\text { completion) } \\
\text { Acceptability by clinical teams and } \\
\text { managers }\end{array}$ \\
\hline
\end{tabular}

Author's

conclusion

score counts' of the standard box would be considered. $^{23-25} 28$

The construct (QoL) that is studied in the current systematic review is a multidimensional concept, therefore, it is important to know whether the instruments accurately represent the multidimensionality of QoL in the individuals living with type 2 diabetes mellitus in India. ${ }^{23-2528}$ Hence, structural validity will be assessed. The RoB checklist for assessing structural validity is composed of four items. The RoB assessment and scoring is similar to content validity, as described above. ${ }^{23-25} 28$

Additional details of assessing RoB for content and structural validity can be found in the COSMIN manual. ${ }^{23-25}$ Five authors (CD, ALJ, AT, JJ and MAH) independently in groups of two, will assess the RoB for each included study and in case of any discrepancy, a third author (SSP) will be contacted. The quality of the evidence will be graded using the Grading of Recommendations Assessment, Development and Evaluation (GRADE) approach. ${ }^{23-29}$ Besides, the quality of our systematic review itself will be evaluated using COSMIN checklist.

\section{Description of studies and analysis}

We will undertake narrative synthesis to analyse the findings. Generic and disease specific QoL instruments will be listed independently in a tabular form with respective dimensions and target population. Furthermore, studies that used instruments developed outside India will be segregated to identify if it were validated among India. Methodological quality of the study will be considered while generating the evidence. We will provide a summary of each criterion for measurement properties of PROM as suggested by Terwee $e t a{ }^{11}{ }^{11}$ Each property will be rated as positive, negative or intermediate. ${ }^{11}$ Through this systematic review, we will provide evidence of the psychometric properties (content validity and internal structure) for each of the PROM included in the study. Furthermore, we will make a recommendation based on the results of the review as suggested by COSMIN manual. ${ }^{23-25}$

Our intent is to identify the QoL instruments used among resident Indian type 2 diabetes population and compare the domains/ construct of these instruments, therefore, the analysis is restricted to narrative synthesis. Additionally, we anticipate heterogeneity in population, context, diabetes complications, types of instruments (generic/ condition specific), construct/ domains being measured and study methods. Considering the aforementioned reasons, we do not intend to undertake statistical pooling.

\section{Patient and public involvement}

We did not have patient or public involvement for designing and writing of this systematic review protocol. However, considering the importance of stakeholders in research we will explore the possibility of including the stakeholders at the final stage of review. We will share the findings of the final review with at least two patients, researchers/professionals working in the field of QoL, and diabetes care management, and social workers. We will get the feedback of stakeholders before submitting the final review to the journal. Considering the resource constraints, we will select the stakeholders affiliated to the authors' institution.

\section{Ethics and dissemination}

Ethical permission is not applicable as this is a systematic review protocol. We intend to present the systematic review findings in a national and/or international conference and publish the findings in a peer-reviewed journal.

\section{Twitter Shradha S Parsekar @ParsekarShrads}

Acknowledgements We are grateful to Manipal Academy of Higher Education (MAHE), Manipal for providing logistic support and infrastructure to carry out this systematic review. We would like to thank the faculty of PHESA, Dept. of Health Information, PSPH, MAHE, Manipal who have helped us in contributing their valuable suggestions and opinions. We would like to express our gratitude to the faculties of the Department of Commerce, MAHE, Manipal for their guidance and encouragement, which has helped us in our work. We extend our gratitude and admiration to Subrata Bhattacharya, Pharma Quant, Kolkata, West Bengal for his worthwhile inputs. We are grateful to Dr. Prachi Pundir, Research Officer, PHESA, PSPH, MAHE, Manipal for proof reading the document. 
Contributors Conceptualisation: $\mathrm{CD}, \mathrm{AR}$ and SSP; writing-original draft preparation: CD, AT, JJ, ALJ and MAH; Writing-review and editing: SSP and Supervision: CD and SSP. All authors read and approved the final manuscript.

Funding The authors have not declared a specific grant for this research from any funding agency in the public, commercial or not-for-profit sectors.

Competing interests None declared.

Patient and public involvement Patients and/or the public were not involved in the design, or conduct, or reporting, or dissemination plans of this research.

Patient consent for publication Not required.

Provenance and peer review Not commissioned; externally peer reviewed.

Open access This is an open access article distributed in accordance with the Creative Commons Attribution Non Commercial (CC BY-NC 4.0) license, which permits others to distribute, remix, adapt, build upon this work non-commercially, and license their derivative works on different terms, provided the original work is properly cited, appropriate credit is given, any changes made indicated, and the use is non-commercial. See: http://creativecommons.org/licenses/by-nc/4.0/.

ORCID iD

Shradha S Parsekar http://orcid.org/0000-0002-8824-9198

\section{REFERENCES}

1 World Health Organization. Diabetes, 2018. Available: https://www. who.int/news-room/fact-sheets/detail/diabetes [Accessed 5 Nov 2019].

2 Diabetes Foundation India, 2013. Available: http://www.diabetesfoun dationindia.org/about.htm

3 John R, Pise S, Chaudhari L, et al. Evaluation of quality of life in type 2 diabetes mellitus patients using quality of life instrument for Indian diabetic patients: a cross-sectional study. J Midlife Health 2019;10:81-8.

4 Ramachandran A. Epidemiology of type 2 diabetes in Indians. $J$ Indian Med Assoc 2002;100:425-7.

5 World Health Organization. Diabetes, 2019. Available: https://www. who.int/health-topics/diabetes\#tab=tab 1 [Accessed 5 Nov 2019].

6 Joshi SR. Diabetes care in India. Ann Glob Health 2015;81:830-8.

7 Trikkalinou A, Papazafiropoulou AK, Melidonis A. Type 2 diabetes and quality of life. World J Diabetes 2017;8:120-9.

8 World Health Organization. Introducing the WHOQOL instruments, 2019. Available: https://www.who.int/healthinfo/survey/whoqolqualityoflife/en/ [Accessed 5 Nov 2019].

9 European Patient's Academy. Measuring health-related quality of life (HRQoL), 2016. Available: https://www.patientsacademy.eu/healthtechnology-assessment/measuring-health-related-quality-life-hrqol/ [Accessed 10 Feb 2020].

10 Fitzpatrick R, Fletcher A, Gore S, et al. Quality of life measures in health care. I: applications and issues in assessment. BMJ 1992;305:1074-7.

11 Terwee CB, Bot SDM, de Boer MR, et al. Quality criteria were proposed for measurement properties of health status questionnaires. J Clin Epidemiol 2007;60:34-42.

12 Kosowski TR, McCarthy C, Reavey PL, et al. A systematic review of patient-reported outcome measures after facial cosmetic surgery and/or nonsurgical facial rejuvenation. Plast Reconstr Surg 2009;123:1819-27.

13 Nagpal J, Kumar A, Kakar S, et al. The development of quality of life Instrument for Indian diabetes patients (QOLID): a validation and reliability study in middle and higher income groups. J Assoc Physicians India 2010;58:295-304.

14 Parik PC, Patel VJ. Health-related quality of life of patients with type 2 diabetes mellitus at a tertiary care hospital in India using EQ 5d 5L. Indian J Endocrinol Metab 2019;23:407-11.

15 PrasannaKumar HR, Mahesh MG, Menon VB, et al. Patient selfreported quality of life assessment in type 2 diabetes mellitus: a pilot study. Niger J Clin Pract 2018;21:343-9.

16 Deswal J, Narang S, Gupta N, et al. To study the impact of diabetic retinopathy on quality of life in Indian diabetic patients. Indian $J$ Ophthalmol 2020;68:848-53.

17 Litwin M. Health-Related Quality of Life. In: Penson DF, Wei JT, eds. Clinical Research Methods for Surgeons [ebook. Totowa, NJ: Humana Press, 2006: 237-51. http://eknygos.Ismuni.lt/springer/587/ 237-251.pdf

18 Hooda SK. Out-pf-pocket payments for healthcare in India: who have affected the most and why? J Health Manage 2017;19:1-15

19 Hart HE, Redekop WK, Bilo HJG, et al. Health related quality of life in patients with type I diabetes mellitus: generic \& disease-specific measurement. Indian J Med Res 2007;125:203-16.

20 Shamseer L, Moher D, Clarke M, et al. Preferred reporting items for systematic review and meta-analysis protocols (PRISMA-P) 2015: elaboration and explanation. BMJ 2015;350:g7647.

21 Terwee CB, Jansma EP, Riphagen II, et al. Development of a methodological PubMed search filter for finding studies on measurement properties of measurement instruments. Qual Life Res 2009;18:1115-23.

22 Mokkink LB, Prinsen CAC, Patrick DL. COSMIN methodology for systematic reviews of patient-reported outcome measures (PROMs): user manual. COSMIN. Available: https://www.cosmin.nl/wp-content/ uploads/COSMIN-syst-review-for-PROMs-manual_version-1_feb2018.pdf

23 Prinsen CAC, Mokkink LB, Bouter LM, et al. COSMIN guideline for systematic reviews of patient-reported outcome measures. Qual Life Res 2018;27:1147-57.

24 Mokkink LB, de Vet HCW, Prinsen CAC, et al. COSMIN risk of bias checklist for systematic reviews of patient-reported outcome measures. Qual Life Res 2018:27:1171-9.

25 Terwee CB, Prinsen CAC, Chiarotto A, et al. COSMIN methodology for evaluating the content validity of patient-reported outcome measures: a Delphi study. Qual Life Res 2018;27:1159-70.

26 Mokkink LB, Terwee CB, Patrick DL, et al. The COSMIN study reached international consensus on taxonomy, terminology, and definitions of measurement properties for health-related patientreported outcomes. J Clin Epidemiol 2010;63:737-45.

27 Terwee CB, Jansma EP, Riphagen II, et al. Development of a methodological PubMed search filter for finding studies on measurement properties of measurement instruments. Qual Life Res 2009;18:1115-23.

28 Terwee CB, Prinsen CAC, Ricci Garotti MG, et al. The quality of systematic reviews of health-related outcome measurement instruments. Qual Life Res 2016;25:767-79.

29 Terwee CB, Mokkink LB, Knol DL, et al. Rating the methodological quality in systematic reviews of studies on measurement properties: a scoring system for the COSMIN checklist. Qual Life Res 2012;21:651-7 